\title{
Consumption of Analeptics and Energizing Drinks to Improve Academic Performance in Students from First to Third Level of the Career of Medicine
}

VII International Congress of

Science, Technology,

Entrepreneurship and

Innovation (SECTEI 2020)

Corresponding Author:

S. Pino

susipinob1955@yahoo.es

Published: 26 August 202

Production and Hosting by

Knowledge E

(c) S. Pino et al. This article is distributed under the terms of the Creative Commons

Attribution License, which permits unrestricted use and redistribution provided that the original author and source are credited.
S OPEN ACCESS
Consumo de Analépticos y Bebidas Energizantes para Mejorar el Rendimiento Académico en Estudiantes de la Carrera de Medicina

\author{
S. Pino ${ }^{1}$, M. López ${ }^{2}$, and J. Viñán ${ }^{3}$ \\ ${ }^{1} \mathrm{ESPOCH}$, Carrera de Medicina, Facultad Salud Pública, Chimborazo, Riobamba, Ecuador \\ ${ }^{2}$ ESPOCH, Unidad de Nivelación y Admisión, Chimborazo, Riobamba, Ecuador \\ ${ }^{3}$ ESPOCH, Carrera de Contabilidad, Facultad Administración de Empresas, Chimborazo, \\ Riobamba, Ecuador
}

\section{Abstract}

Central nervous system stimulant drugs are frequently used by college students as factors to alleviate fatigue, maintain wakefulness, and stimulate cognitive abilities, which has become a problem because the physical or emotional consequences that may occur are not considered. produce. At the time of marketing these products they do not keep any type of control, it is freely distributed to the general public. Objective: to analyze how the consumption of analeptics and energy drinks affects the academic performance of students. Methods: An explanatory, cross-sectional, quantitative-qualitative study was carried out, to obtain data, the survey was applied to first to third level students of the Medicine School of the Polytechnic School of Chimborazo (ESPOCH). Results: From the compilation of the information it can be determined that $65 \%$ of the students use stimulants to increase their performance, without considering the consequences such as palpitations, insomnia, headache, tremor, vomiting, gastrointestinal discomfort as well as affections to different organs and body systems. Bottom Line: The cognitive effects of stimulants commonly bring about changes in performance by accelerating the production of dopamine in the brain, which is a neurotransmitter that is related to attention, pleasure, memory and performance. Adverse effects can vary from mild to very serious, causing irritability, headache, arrhythmias, hypertension, dizziness, and lesser degrees such as difficulty in bowel movement, itching or skin rash, among others.

Keywords: analleptic, stimuli, effects, academic performance.

\section{Resumen}

Los estudiantes universitarios consumen con frecuencia los fármacos estimulantes del sistema nervioso central como factores para aliviar la fatiga, mantener la vigilia y estimular las capacidades cognitivas, lo cual se ha convertido en un problema, porque no se consideran las consecuencias físicas o emocionales que se pueden producir. Al momento de comercializar estos productos no guardan algún tipo de control, es de libre distribución al público general. Objetivo: analizar como el consumo de analépticos y bebidas energizantes incide en el 
rendimiento académico de los estudiantes. Métodos: Se realizó un estudio explicativo de corte transversal, de tipo cuanti- cualitativo, para la obtención de datos se aplicó la encuesta a los estudiantes de primer a tercer nivel de la Carrera de Medicina de la Escuela Superior Politécnica de Chimborazo (ESPOCH). Resultados: De la recopilación de la información se puede determinar que un $65 \%$ de los estudiantes utilizan los estimulantes para elevar su rendimiento, sin considerar las consecuencias como palpitaciones, insomnio, cefalea, temblor, vómito, molestias gastrointestinales así como afecciones a distintos órganos y sistemas del cuerpo. Conclusión: Los efectos cognitivos de los estimulantes, comúnmente aportan cambios en el rendimiento al acelerar la producción de dopamina en el cerebro, que es un neurotransmisor que se relaciona con la atención, el placer, la memoria y el rendimiento. Los efectos adversos pueden variar de leves a muy graves causando irritabilidad, dolor de cabeza, arritmias, hipertensión, mareos y los de menor grado como dificultad para la evacuación intestinal, picazón o erupción en la piel, entre otras.

Palabras Clave: analépticos, estímulos, efectos, rendimiento académico.

\section{Introducción}

El trabajo de investigación permite describir la situación de los estudiantes de primero a tercer nivel de la carrera de Medicina de la Escuela Superior Politécnica de Chimborazo frente al consumo de analépticos y bebidas energizantes, productos que son utilizados como una forma de aliviar la fatiga, mantenerse agiles mentalmente, poder contrarrestar el sueño y estimular las capacidades cognitivas de manera que tengan un mejor rendimiento académico, frente a las exigencias que demanda el estudio en esta carrera, por ser una de las más largas y con un alto grado de preparación académica, porque en las manos de los futuros médicos se encontrará la vida de muchas personas. Durante la etapa académica los estudiantes deben formarse con una amplia y compleja malla curricular, siendo el estudio diario de vital importancia. Por lo cual, los estudiantes buscan tener el mejor rendimiento posible, incluso muchas de las veces sacrifican el sueño para poder estudiar un poco más.

El consumir estimulantes para el sistema nervioso central (SNC), se ha convertido en una actividad habitual porque la venta no guarda algún tipo de control al momento de ser comercializadas, es de libre distribución al público general, por lo cual se ha hecho común su uso evadiendo e ignorando las consecuencias futuras que inducen a efectos neurológicos, fisiológicos, psicológicos y emocionales debido a su capacidad de modificar la acción de los neurotransmisores en el cerebro.

Del análisis del contexto del problema de investigación se puede determinar que según resultados de la investigación, en Nueva York, Turquía, y Canadá son poblaciones 
en las cuales aproximadamente el $34,80 \%$ de sus estudiantes consumen estos fármacos con el objetivo de lograr adquirir un alto rendimiento académico.

En Latinoamérica, el 64,90\% de personas han ingerido bebidas energizantes, de ellos $87,60 \%$ las han mesclado con alcohol; los consumidores principales son personas entre 14 y 25 años aduciendo que el consumo ayuda a proporcionar energía y mantenimiento de la vigilia, facilitación de la ebriedad y vinculación social.

Se han podido evidenciar también investigaciones a nivel nacional, tal es el caso de la Universidad Católica del Ecuador en donde 293 estudiantes fueron encuestados y se determinó que la prevalencia general de consumo de psico estimulantes es del $62.4 \%$. La edad promedio fue de 20.9 años, el $45.3 \%$ de los participantes correspondieron al sexo masculino y el $54.6 \%$ al sexo femenino. En términos generales la sustancia con mayor prevalencia de consumo fue el café con un $52 \%$ y dentro de los fármacos el modafinilo con un $14 \%$.

Por lo expuesto se determina la necesidad de identificar ¿cuáles son los factores que intervienen en el consumo de analépticos para elevar el rendimiento académico de los estudiantes de la carrera de medicina de la ESPOCH?

Se justifica la investigación para dar respuesta a la pregunta de investigación y además lograr el objetivo que se propone al realizar el presente trabajo como es el analizar como el consumo de analépticos y bebidas energizantes incide en el rendimiento académico de los estudiantes de la carrera de Medicina en los primeros niveles.

\section{Metodología}

\subsection{Diseño}

Se realizó un estudio explicativo de corte transversal, de tipo cuanti cualitativo, los datos se obtuvieron en las dependencias de la carrera de medicina de la ESPOCH con la aplicación de la encuesta a los estudiantes de primero a tercer nivel, que previamente fue validada para garantizar la calidad de la información que se recopila. Además la para la fundamentación teórica del tema de investigación, se realizó una revisión rigurosa del material bibliográfico disponible.

\subsection{Población y muestra}

La población en el presente estudio constituyen los 570 estudiantes matriculados de primero al tercer nivel de la Carrera de Medicina de la ESPOCH en el periodo académico marzo - julio de 2019 como se registra en: 


\section{Table 1}

Número de estudiantes por semestre.

Niveles
Primer semestre
Segundo semestre
Tercer semestre
Total

Número de estudiantes

261

171

97

570

Fuente: Secretaría de la Carrera de Medicina.

Elaborado por: S. Pino, M J, López, J. Viñán.

Muestra: Se calculó en función de la aplicación de la formula estadística de muestreo probabilístico debido a que todos los estudiantes tienen la probabilidad de ser elegidos con un $95 \%$ de nivel de confianza por lo tanto se realiza el cálculo en base a la siguiente formula:

$$
n=\frac{Z^{2} p * q * N}{Z^{2} * p * q \& N e^{2}}
$$

Fórmula:

Valores a estimar

n: Tamaño de la muestra;

N: Población total 570;

e: Error de estimación 5\% = 0.05;

z: Nivel de confianza 95\% $(1,96)$;

p: Probabilidad a favor $0.5 ; y$

q: Probabilidad en contra $\mathbf{0 . 5}$.

La muestra se obtuvo a través del respectivo cálculo con $\mathbf{2 3 0}$ estudiantes, que fueron encuestados para obtener la información de manera directa. Para elegir a los encuestados se empleó un muestreo aleatorio simple ya que los estudiantes fueron elegidos al azar.

\subsection{Procedimientos para validar la calidad de los datos obtenidos}

La información recopilada se procede a procesarla con ayuda de Excel y mediante pruebas de significación estadística se analizaron los datos, que se resumen en las tablas y gráficos para la mejor comprensión.

La información que se recepta incluye: edad, sexo, nivel que cursa, hábitos de estudio, complejidad de la carrera, empleo de tiempo para estudiar, información de los analépticos y bebidas energizantes y los efectos que producen. Las estrategias básicas son la codificación de frases y expresiones, la formación de categorías que 
hacen referencia al comportamiento social estudiado, la reflexión y análisis sobre estas categorías, así como sus relaciones para obtener un esquema teórico.

\section{Resultados y Discusión}

Para el estudio del presente trabajo se aplicó la encuesta consistente en 10 preguntas a 230 estudiantes de primero al tercer nivel de la carrera de medicina de la ESPOCH.

A continuación se presentan los resultados más relevantes que contribuyen a determinar las causas y las consecuencias del consumo de analépticos y bebidas energizantes.

\section{Table 2}

Frecuencia de consumos analépticos clasificados por sexo de los estudiantes.

\begin{tabular}{|c|c|c|c|c|}
\hline $\begin{array}{l}\text { Frecuencia del } \\
\text { consumo }\end{array}$ & Femenino & $\%$ & Masculino & $\%$ \\
\hline Nunca & 78 & 40,63 & 12 & 31,58 \\
\hline $\begin{array}{l}\text { Una vez a la } \\
\text { semana }\end{array}$ & 52 & 27,08 & 11 & 28,95 \\
\hline Una vez al mes & 44 & 22,92 & 9 & 23,68 \\
\hline $\begin{array}{l}\text { Dos o más veces } \\
\text { al mes }\end{array}$ & 18 & 9,38 & 6 & 15,79 \\
\hline Total & 192 & 100 & 38 & 100 \\
\hline
\end{tabular}

Fuente: Encuesta a estudiantes.

Elaborado por: S. Pino, M J, López, J. Viñán.

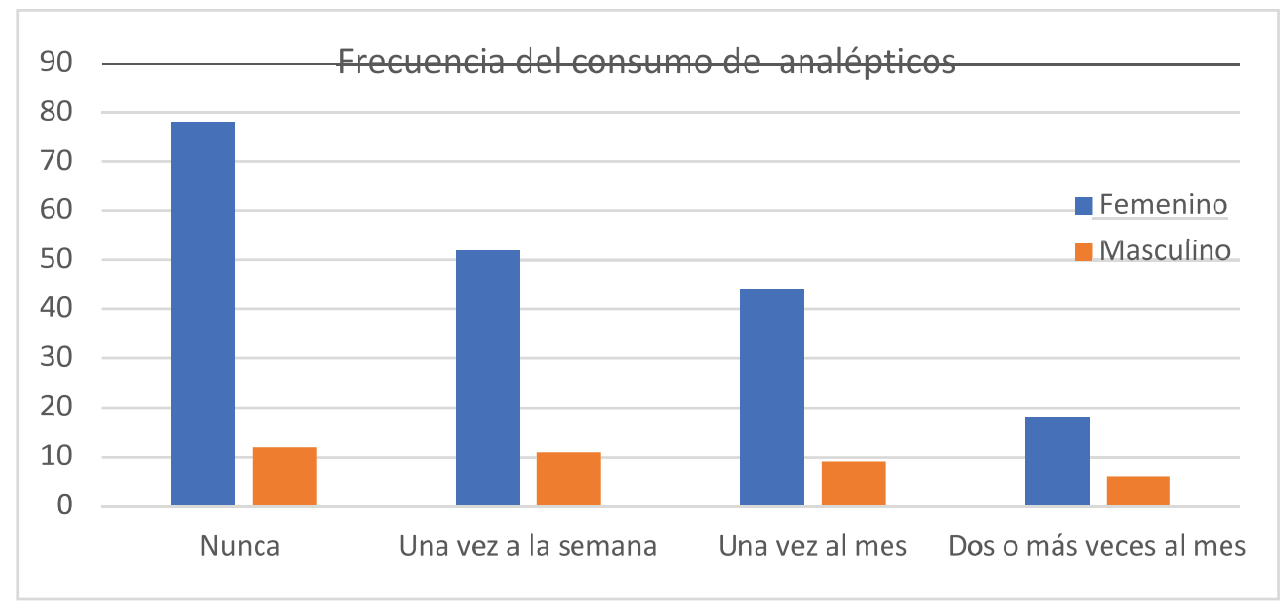

\section{Figure 1}

Fuente: Tabla 2. Elaborado por: S. Pino, M J, López, J. Viñán.

Análisis e interpretación: Del total de las encuestas aplicadas, el 40,63\% del sexo femenino indican que nunca han consumido alguna sustancia que estimule directamente el SNC, mientras que el 9,38\% han consumido dos o más veces al mes estas 
sustancias, en tanto que, el $31,58 \%$ de sexo masculino menciona que nunca han consumido, mientras que el $15,79 \%$ considera que lo han hecho dos o más veces al mes. Por lo que se puede determinar que en un porcentaje mayor a la mitad de los estudiantes de ambos sexos han consumido analépticos con el fin de mejorar su estado físico para elevar el rendimiento académico en razón de que en los primeros niveles deben garantizar la permanencia en la carrera.

\section{Table 3}

Efedrinas utilizadas para estimular el SNC mantener la vigilia.

\begin{tabular}{ll|l|l|l|l|l}
\hline $\begin{array}{l}\text { Efedrinas que } \\
\text { utilizan }\end{array}$ & Primero & $\%$ & Segundo & $\%$ & Tercero & $\%$ \\
\hline $\begin{array}{l}\text { Antefetaminas en } \\
\text { comprimidos }\end{array}$ & 0 & 0 & 10 & 6,67 & 0 & 0 \\
\hline $\begin{array}{l}\text { Fenproporex } \\
\text { Fenfluramina }\end{array}$ & 1 & 3,03 & 2 & 1,33 & 1 & 2,13 \\
\hline Metilfenidato & 0 & 3,03 & 5 & 3,33 & 1 & 2,13 \\
\hline Carim & 10 & 0 & 17 & 11,33 & 1 & 2,13 \\
\hline Alertex & 16 & 30,3 & 21 & 14 & 17 & 36,17 \\
\hline Ninguno & 5 & 48,48 & 25 & 16,67 & 12 & 25,53 \\
\hline Total & $\mathbf{3 3}$ & 15,15 & 70 & 46,67 & 15 & 31,91 \\
\hline
\end{tabular}

Fuente: Encuesta a estudiantes.

Elaborado por: S. Pino, M J, López, J. Viñán.

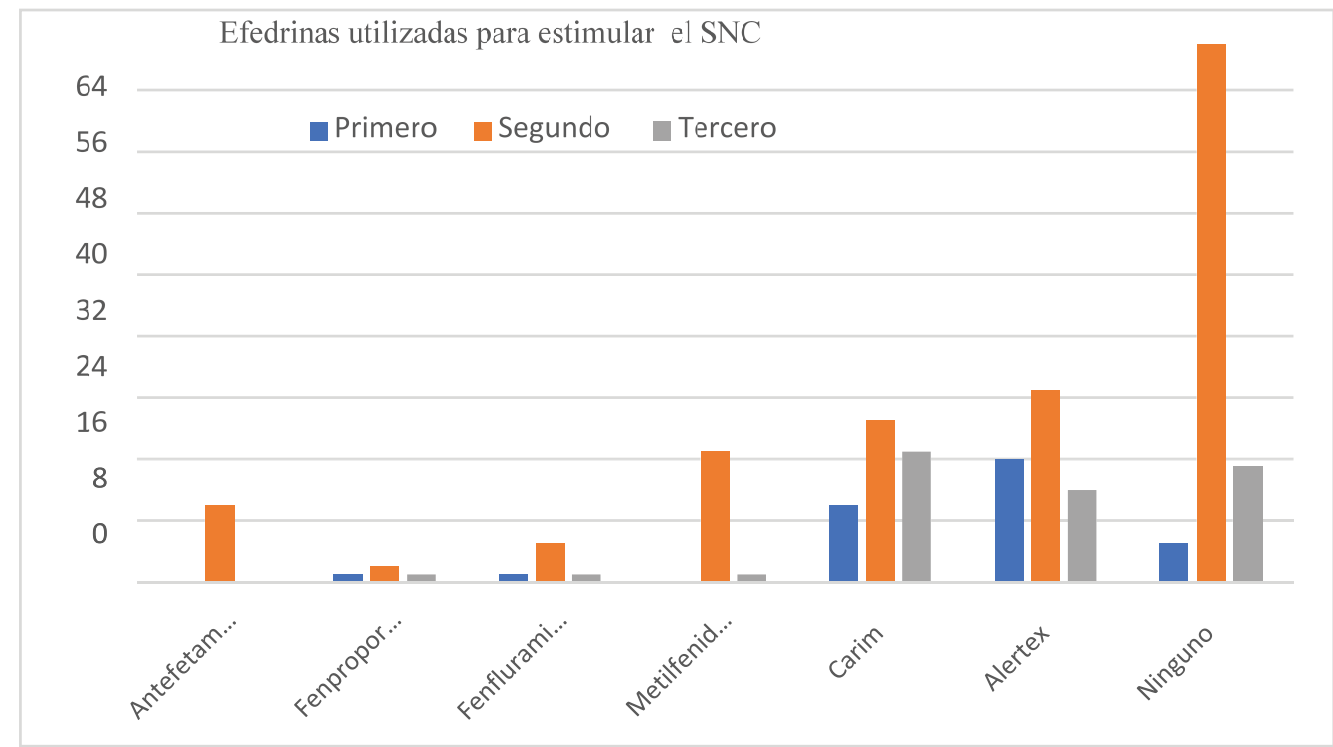

Figure 2

Fuente: Tabla 3. Elaborado por: S. Pino, M.J, López, J. Viñán.

Análisis e interpretación: De los estudiantes encuestados, el 48,48\% del primer nivel consumen alertex, en tanto que en segundo nivel el $46,67 \%$ manifiesta que no consume 
ninguna efedrina, respecto al tercer nivel el 36,67\% indican que utilizan carim como estimulante del SNC. Por lo que se puede determinar que la mayoría de los estudiantes que consumen efedrinas prefieren el carim y alertex y que en el primero y segundo nivel es donde más utilizan los estimulantes del SNC para mantenerse despiertos y poder preparar las pruebas, cumplir con los trabajos y lograr un buen rendimiento académico.

\section{Table 4}

Causa del consumo de analépticos

\begin{tabular}{l|l|l|l|l|l} 
& Si & \% & No. & $\%$ & Total \\
\hline Estudiar & 58 & 39,19 & 15 & 18,29 & 73 \\
\hline Hacer deportes & 48 & 32,43 & 36 & 43,90 & 84 \\
\hline Suplemento vitamínico & 8 & 5,41 & 8 & 9,76 & 16 \\
\hline Refrescante de la sed & 7 & 4,73 & 0 & 0,00 & 7 \\
\hline Todas & 22 & 14,86 & 11 & 13,41 & 33 \\
Ninguna & 5 & 3,38 & 12 & 14,63 & 17 \\
\hline Total & $\mathbf{1 4 8}$ & $\mathbf{1 0 0 , 0 0}$ & $\mathbf{8 2}$ & $\mathbf{1 0 0 , 0 0}$ & $\mathbf{2 3 0}$
\end{tabular}

Fuente: Encuesta a estudiantes. Elaborado por: S. Pino, M J, López, J. Viñán.

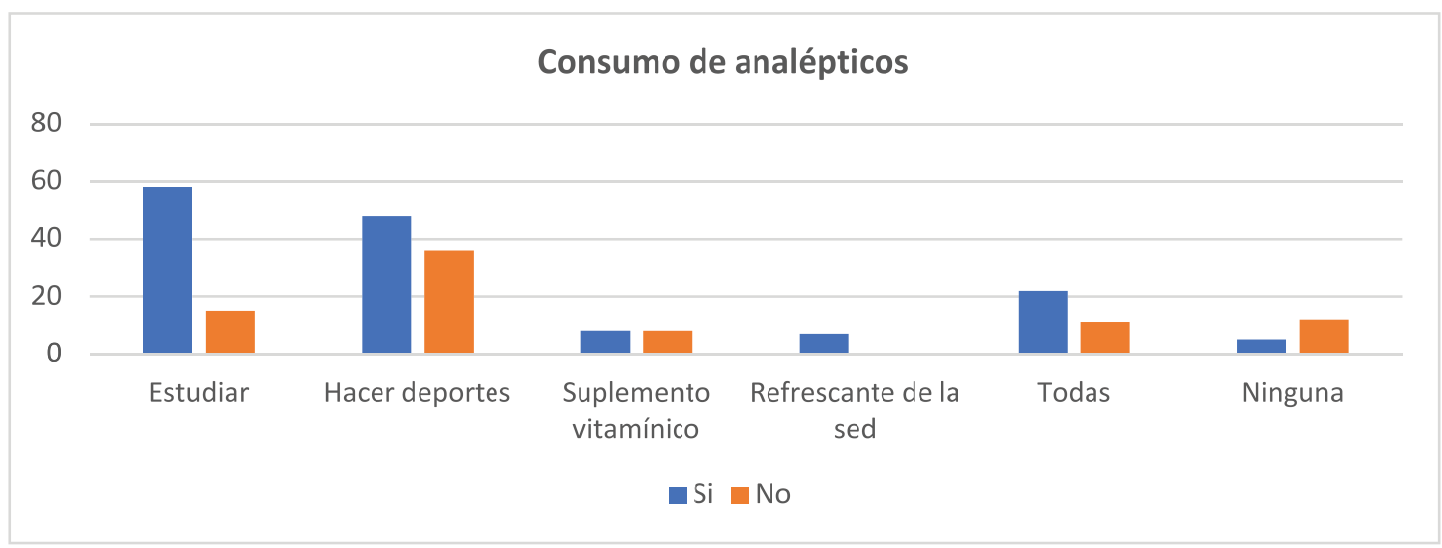

Figure 3

Fuente: Tabla 4. Elaborado por: S. Pino, M.J, López, J. Viñá.

Análisis e interpretación: De los estudiantes encuestados que indican que en el presente período académico si han consumido analépticos, el 39,19\% manifiestan que lo hacen para estudiar, mientras que el 3,38\% indican que no encuentran ninguna razón. Por otra parte los que manifiestan que no han consumido en un $43,90 \%$ indican que es para hacer deporte frente al 9,76 consideran que es un suplemento vitamínico, por lo expresado se puede considerar que la mayoría indican que el consumo de estas sustancias es de utilidad y sirve para favorecer la conservación de la energía frente a las actividades que como estudiantes tienen que cumplir.

Análisis e interpretación: De los resultados para determinar el consumo de bebidas energizantes el $65,22 \%$ indican que si consumen en tanto que en el 34,78\% manifiestan 


\section{Table 5}

Bebidas energizantes que consumen.

\begin{tabular}{|c|c|c|c|}
\hline & Todoslosdías & $\%$ & $\begin{array}{l}\text { Unavez } \\
\text { Semana }\end{array}$ \\
\hline $\begin{array}{l}\text { Redbull } \\
\text { Mosnte }\end{array}$ & 2 & 4,26 & 4 \\
\hline $\mathbf{r}$ & 2 & 4,26 & 6 \\
\hline $\begin{array}{l}\text { Shark } \\
\text { Coca }\end{array}$ & 2 & 4,26 & 2 \\
\hline Cola & 9 & 19,15 & 15 \\
\hline Café & 18 & 38,30 & 16 \\
\hline $\begin{array}{l}220 \text { V } \\
\text { Powera }\end{array}$ & 4 & 8,51 & 1 \\
\hline $\begin{array}{l}\text { de } \\
\text { Gatora }\end{array}$ & 4 & 8,51 & 8 \\
\hline de Vive & 1 & 2,13 & 6 \\
\hline 100 & 5 & 10,64 & 5 \\
\hline Otro & 0 & 0,00 & 1 \\
\hline Total & 47 & 100,00 & 64 \\
\hline
\end{tabular}

\begin{tabular}{|l|l|}
\hline$\%$ & $\begin{array}{l}\text { Dos } \\
\text { amás } \\
\text { veces a } \\
\text { la } \\
\text { semana }\end{array}$ \\
\hline 6,25 & 14 \\
\hline 9,38 & 6 \\
\hline 3,13 & 0 \\
\hline 23,44 & 1 \\
\hline 25,00 & 6 \\
\hline 1,56 & 2 \\
\hline 12,50 & 5 \\
\hline 9,38 & 3 \\
\hline 7,81 & 1 \\
\hline 1,56 & 1 \\
\hline 100,00 & 39 \\
\hline
\end{tabular}

\begin{tabular}{|l|l|}
\hline$\%$ & Nunca \\
\hline 35,90 & 2 \\
\hline 15,38 & 5 \\
\hline 0,00 & 0 \\
\hline 2,56 & 4 \\
\hline 15,38 & 9 \\
\hline 5,13 & 0 \\
\hline 12,82 & 2 \\
\hline 7,69 & 0 \\
\hline 2,56 & 0 \\
\hline 2,56 & 58 \\
\hline 100,00 & 80 \\
\hline
\end{tabular}

\begin{tabular}{|l|l|}
\hline$\%$ & Total \\
\hline 2,50 & 22 \\
\hline 6,25 & 19 \\
\hline 0,00 & 4 \\
\hline 5,00 & 29 \\
\hline 11,25 & 49 \\
\hline 0,00 & 7 \\
\hline 2,50 & 19 \\
\hline 0,00 & 10 \\
\hline 0,00 & 11 \\
\hline 72,50 & 60 \\
\hline 100,00 & 230 \\
\hline
\end{tabular}

Fuente: Encuesta a estudiantes.

Elaborado por: S. Pino, M J, López, J. Viñán.

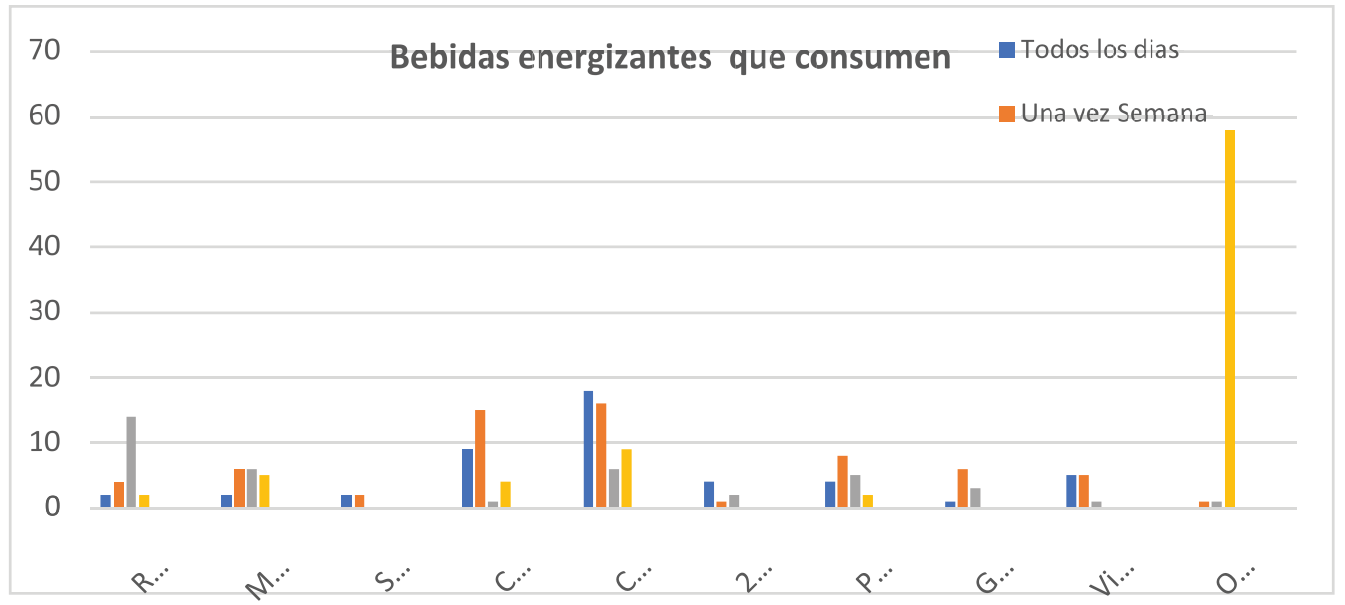

Figure 4

Fuente: Tabla 5.

Elaborado por: S. Pino, M.J, López, J. Viñán.

que nunca han consumido ninguno de los energizantes, por otra parte la bebida que más se consume es el café y lo hacen todos los días, pero también hay una considerable preferencia por la Coca Cola, resultando alarmante por el alto contenido químico que contiene esta bebida. Son de menor preferencia el redbull, shark y Monster debido a la 
poca comercialización de estos productos en el mercado de venta y tan solo el 2,13\% de los estudiantes consume Gatorade todos los días por el precio elevado.

\section{Resultados y Discusión}

\subsection{Analépticos}

La palabra Analéptico se define como aquel fármaco que aumentan la actividad funcional de las células tejidos y órganos a fin de provocar estimulación en el Sistema Nervioso Central (SNC) y que están indicados en el tratamiento de las depresiones provocadas por estados de desequilibrio. De esta forma los Psicoanalépticos son sustancias que aceleran la actividad del sistema nervioso y estimulan el humor, aumentando los niveles de actividad cognitiva y motriz, reforzando la vigilia, el estado de alerta y atención [1].

También son llamados psicoestimulantes produciendo efectos psicoactivos, cuyo principal efecto es el de producir un aumento de la activación cerebral, produce por norma general una alteración y aceleración de la actividad y el estado de ánimo [2].

El analéptico actúa principalmente sobre el centro de control cardíaco y el centro de control respiratorio del encéfalo; son analéptico la adrenalina, la cafeína, el alcanfor, la efedrina y la estricnina.

\subsection{Efedrinas}

Adrenérgico muy activo se constituye en un potente estimulante del sistema nervioso simpático, utilizado con fines médicos para tratar problemas de salud como la depresión y narcolepsia. Actúa de manera similar que la anfetamina su consumo ayuda a reducir la sensación de fatiga e incrementar el estado de alerta, puede aumentar el metabolismo en los tejidos, suele usarse como estimulante, descongestionante, potenciadora de la concentración. En la actualidad su uso se ha extendido de forma recreativa e ilegal debido a su producción en laboratorios clandestinos. Este estimulante presenta rasgos históricos importantes, se resumen así [3]:

La efedrina fue sintetizada en 1887 por Lazar Edeleanu, un químico rumano. Su utilización empezó en 1920, durante la segunda Guerra Mundial, época en la que era común que los miembros de la fuerza área utilizaran la sustancia para poder estar alertas y evitar el cansancio. En 1927 comenzó su comercialización, debido a que ésta podía dilatar los bronquios, contraer vasos sanguíneos y elevar la presión arterial. En 1938 empezó a venderse la metanfetamina (sintetizada en Japón en el año 1919) y posteriormente, en 1954 empezó a comercializarse el metilfenidato. En 1971, debido al abuso y adicción de la sustancia, ésta fue controlada por la comunidad internacional. 
El uso militar de las anfetaminas fue importante durante la Segunda Guerra Mundial, tanto de los ejércitos aliados (Bencedrina sulfato), como del ejército alemán y japonés (metanfetamina). Los pilotos de los bombarderos también utilizaban con fines estimuladores para combatir el cansancio e incrementar el nivel de atención, existían normas para su utilización, pero no se respetaban [4].

Hasta que en 1983 se reguló su venta en farmacias, el consumo de anfetaminas fue muy importante, ya que, hasta el día de hoy, entre las poblaciones que optan por su consumo están desde de estudiantes que intentan aguantar las noches sin dormir con fines académicos hasta camioneros que hacían largas travesías y amas de casa a quienes sobrecargaban las tareas del hogar.

Estos fármacos pueden ser legales o ilegales. Se convierten en legales cuando es recetada por un médico y se utilizan para tratar problemas de salud tales como obesidad o trastorno de hiperactividad por déficit de atención (THDA), sin embargo, puede producir adicción las más utilizadas son: Fenproporex, Fenfluramina, Metilfenidato, Carim, Alertex. Y son ilegales cuando se usan sin receta médica es decir indiscriminadamente con el fin de ser usados como una droga [5].

Pueden ocasionar taquicardia, arritmias cardiacas, angina de pecho, sofoco, sudoración, acné, náuseas, pérdida de apetito, aumento en la excreción de orina debido al aumento en el flujo de la sangre, inquietud, insomnio, euforia, ilusiones, hostilidad, pánico y agitación que son factores poco favorables para los estudiantes de la carrera de medicina.

\subsection{Antidepresivos}

Son psicoanalépticos utilizados para tratar la depresión, con el fin de reducir los síntomas pues no combaten, actúan como estimulante del sistema nervioso central. Ciertas sustancias químicas del cerebro denominadas «neurotransmisores» se relacionan con la depresión, en especial, la serotonina, la norepinefrina y la dopamina. La mayoría de los antidepresivos alivian la depresión al afectar estos neurotransmisores de manera ligeramente diferente. Existen muchas clases de antidepresivos, entre ellos [6]:

(i) Inhibidores de recaptación de serotonina y norepinefrina, IRSN (SNRI en inglés)

(iii) Inhibidores de recaptación de norepinefrina y dopamina, IRND (NDRI en inglés)

(iii) Inhibidores de monoamino oxidasa, IMAO (MAOI en inglés)

Los Inhibidores selectivos son los más importantes por la recaptación de serotonina, cuando una persona no tomó nunca antidepresivos, el primer tipo de medicamento que el médico recomienda es un ISRS. Estos medicamentos tienden a tener menos efectos secundarios que otros antidepresivos. 
Se mencionan algunos de los efectos secundarios que pueden ser por causa de los ISRS son:

(i) Resequedad en la boca

(ii) Náuseas

(iii) Nerviosismo

(iv) Insomnio (dificultad para conciliar el sueño)

(v) Problemas sexuales

(vi) Dolor de cabeza

Los estudiantes consumen de forma indiscriminada los psicoanalépticos con el objetivo de evitar conciliar el sueño y alcanzar a revisar el contenido de las asignaturas de mane4ra preferencial en época de exámenes en dosis más bajas que resulta ser lo justo y necesario para provocar y generar el estímulo, pues uno de sus efectos secundarios es la dificultad para dormir e insomnio. Entre los más usados están: Actan, Amirel, Bectam, Axone, Sertralina.

\subsection{Anticolinérgicos}

Los anticolinérgicos son sustancias, naturales o sintetizadas, que actúan inhibiendo la actividad de la acetilcolina (sustancia química que transmite impulsos) en el sistema nervioso central y periférico. Son fármacos que actúan bloqueando alguno de los receptores de la acetilcolina, que son de dos tipos: muscarínicos y nicotínicos [7].

En función al receptor que busquen bloquear se clasifican en dos tipos:

(i) Antagonistas muscarínicos: Localizados en los de origen natural, destacan la atropina y la escopolamina. Entre los sintéticos: la atropina, brademil, homatropina, benzotropina, telenzepina, viadil y darifenacina.

(ii) Antagonistas nicotínicos: Entre ellos figuran: el curare, la succinilcolina, la alfabungarotoxina y la galamina.

\subsection{Bebidas energizantes}

Un número elevado de estudiantes universitarios presentan un alto consumo de bebidas energizantes, por los niveles de exigencia que se requiere en el ambiente académico, como entrega de trabajos de investigación, cantidad de deberes y exámenes. Los productos energizantes favorecen el aumento de los niveles de energía, evitan el sueño y proporcionan un incremento de la condición física o estado 
de salud, por lo que permite mantenerse en un mejor estado para aumentar la habilidad mental y elevar el rendimiento académico.

Las bebidas que se consumen con mayor frecuencia son: el café, Coca Cola y Pepsi y respecto a las bebidas categorizadas como energizantes, el Red Bull es la de mayor consumo, bebidas que gracias al márquetin publicitario han logrado alcanzar una elevada popularidad, por las propiedades estimulantes y las características que presentan las mismas. El consumo de bebidas energéticas se mantiene elevado a pesar de que producen efectos secundarios [8].

Las bebidas energizantes son consideradas por el efecto de estimulación mental, tienen altos niveles de cafeína, taurina que eliminan la sensación de agotamiento de la persona que las consume, en tanto que las bebidas hidratantes no contienen cafeína y si la contiene se encuentran en niveles bajos.

Algunos autores consideran que la cafeína es una sustancia adictiva, que al igual que otras sustancias psicoactivas produce tolerancia, síndrome de abstinencia y persistencia del uso a pesar del daño. Claro está que la cafeína provoca un estímulo al cerebro, al disminuir la acción de la adenosina, un transmisor nervioso que produce calma genera una sensación de mayor energía durante algunas horas.

Las bebidas energizantes contienen gran cantidad de carbohidratos (sacarosa, glucosa), aminoácidos, proteínas, vitaminas del complejo B (B1, B2, B6, B12, vitamina C, Niacina, ácido pantotéico), metilxantinas tales como la cafeína, teofilina y teobromina, sustancias derivadas de hierbas como el extracto de guaraná y ginseng. Además, tienen otras sustancias en cantidades inferiores como inositol, carnitina, pantoteato de calcio, biotina, glucoronolactona y ácido cítrico que ayudan a eliminar la sensación de agotamiento de la persona que consume.

\subsubsection{Efectos producidos}

Investigaciones realizadas en ratones muestran la gran desventaja que puede producir la homeostasis corporal por el uso prolongado de estas bebidas y tan solo un pequeño porcentaje muestra la ventaja del consumir estos productos.

De las ventajas respecto al factor Neuropsiquiátrico se pueden evidenciarse efectos positivos a nivel actitudinal, mejora en el desempeño de tareas manuales y cognoscitivas por las propiedades estimulantes que poseen las metilxantinas.

$\mathrm{Si}$ el consumo de estas bebidas es frecuente causa afecciones a distintos órganos y sistemas del cuerpo los más frecuentes son:

- Cardiovascular: Elevación de la presión arterial, palpitaciones rápidas del corazón (Taquicardia). 
- Intoxicación: Los síntomas más frecuentes son nerviosismo, ansiedad, temblor, malestar gastrointestinal y en algunos casos la muerte. En el caso de los niños y adolescentes el riesgo de intoxicación al ingerir una bebida energizante o estimulante es elevado a comparación de una persona adulta.

- Síndrome de abstinencia: Caracterizado por presencia de cefalea, náuseas, irritabilidad y depresión. Dicha sintomatología se inicia 12-24 hr después de suspender el consumo de cafeína o bebidas energizantes y puede tardar en resolverse hasta una semana posterior al consumo [9].

Los efectos fisiológicos y adversos de las bebidas energizantes se observan en los cambios en el rendimiento demostrado que combinando la cafeína y taurina provoca una disminución en el tiempo de reacción al contrastar con el placebo, por otro lado, no se encontraron efectos significativos sobre la memoria a corto plazo. En consecuencia, entre los efectos adversos que se presencian posterior a la ingesta de dichas bebidas son las palpitaciones, seguido de insomnio, cefalea, temblor, náusea, vómito, molestias gastrointestinales y efectos varios en el SNC [10].

\section{Table 6}

Tomado de la tesis Consumo de psico estimulantes para aumentar el rendimiento en el autoestudio.

\begin{tabular}{|c|c|c|c|c|}
\hline $\begin{array}{l}\text { Potencia } \\
\text { dor } \\
\text { cognitivo }\end{array}$ & $\begin{array}{l}\text { Mecanismo } \\
\text { modulador }\end{array}$ & $\begin{array}{l}\text { Mejoramiento } \\
\text { de las funciones } \\
\text { cognitivas }\end{array}$ & $\begin{array}{l}\text { Sistemas } \\
\text { cerebrales } \\
\text { conocidos más } \\
\text { afectados }\end{array}$ & $\begin{array}{l}\text { Uso clínico } \\
\text { recomendado }\end{array}$ \\
\hline $\begin{array}{l}\text { Anfetamin } \\
\text { a }\end{array}$ & $\begin{array}{lr}\text { Inhibidor de } & \text { la } \\
\text { receptación } & \text { de } \\
\text { dopamina } & \text { y } \\
\text { noradrenalina } & \end{array}$ & $\begin{array}{l}\text { Inhibe la } \\
\text { respuesta a la } \\
\text { memoria de } \\
\text { trabajo, atención } \\
\text { y vigilia }\end{array}$ & $\begin{array}{l}\text { Sistema de } \\
\text { atención } \\
\text { frontoparietal } \\
\text { estriado }\end{array}$ & $\begin{array}{l}\text { Trastorno } \\
\text { déficit de } \\
\text { atención con } \\
\text { hiperactividad }\end{array}$ \\
\hline Cafeína & $\begin{array}{ll}\text { Antagonista } & \text { no } \\
\text { selectivo de } & \text { los } \\
\text { receptores } & \text { de } \\
\text { adenosina } & \end{array}$ & $\begin{array}{l}\text { Vigilia, memoria } \\
\text { de trabajo, } \\
\text { aprendizaje } \\
\text { incidental }\end{array}$ & $\begin{array}{ll}\text { Sistema de } \\
\text { atención de } \\
\text { lóbulo frontal }\end{array}$ & - \\
\hline Modafinilo & $\begin{array}{l}\text { No se conoce, pero } \\
\text { sus probables } \\
\text { efectos están } \\
\text { relacionados con la } \\
\text { dopamina, } \\
\text { noradrenalina y } \\
\text { orexina. }\end{array}$ & $\begin{array}{l}\text { Memoria de } \\
\text { trabajo, memoria } \\
\text { episódica } \\
\text { atención }\end{array}$ & $\begin{array}{l}\text { Sistema de } \\
\text { atención lóbulo } \\
\text { frontal }\end{array}$ & $\begin{array}{l}\text { Agente } \\
\text { promotor } \\
\text { despertar }\end{array}$ \\
\hline
\end{tabular}

Fuente: Tesis para optar al título de Médico General y Cirujano (2013; p. 25).

Del consumo de la cafeína no se conocen con precisión los porcentajes seguros, de la información consultada se recomienda que en un adulto sano podrían ser hasta de 400 mg por día. Sin embargo se puede observar que, luego de la ingesta de 
bebidas que contiene cafeína y otros energizantes, algunas personas han ingresado a establecimientos de salud ya sea por arritmias, convulsiones, agitación, agresividad e ideación suicida, principalmente cuando se acompaña dichas bebidas con el uso de etanol [11].

Algunos informes de casos clínicos han presenciado efectos adversos como consecuencia de la ingesta crónica de bebidas energizantes psíquicas, así el de accidente cerebrovascular isquémico y convulsiones, síndrome de QT largo e infarto agudo de miocardio; también se han podido evidenciar efectos adversos de los ingredientes de estas bebidas, tales como la taurina que se asocian con taquicardia, agitación, sangrado, alteración del estado de conciencia y convulsiones tónico clónicas [12].

\subsubsection{Efectos fisiológicos y adversos de los psicoanalépticos}

Si bien se conoce los psicoanalépticos aceleran la producción de dopamina en el cerebro, que es un neurotransmisor que se relaciona con la atención, el placer, la memoria y el movimiento, entre otras. En dosis elevadas alteran la comunicación de las células del cerebro provocando efectos adversos tales como el riesgo de adicción o euforia.

Los efectos fisiológicos varían de leves a muy graves siendo los de mayor grado la irritabilidad, dolor de cabeza, palpitaciones, arritmias, hipertensión, mareos; entre otros y los de menor grado tales como: dificultad para la evacuación intestinal, boca seca, sabor desagradable, enrojecimiento de la piel, urticaria, ronchas, picazón o erupción en la piel, entre otras.

Sin embargo la mezcla de psicoanalépticos con alcohol pude ser más perjudiciales con resultados es una serie de consecuencias para la salud como las arritmias cardiacas fatales [13].

Por el contrario, existe gran cantidad de información que indica que las bebidas psíquicas y los psicoanalépticos presentan beneficios al estudiante sobre actividades que son esenciales en el aumento inmediato del rendimiento cognitivo o físico, sobre todo en situaciones grandes de cansancio.

Del mismo modo la mayor parte de estos efectos beneficiosos que presentan que pueden ser atribuidos a las bebidas psíquicas y los psicoanalépticos parecen estar especialmente vinculados con la existencia de cafeína y no tanto con el resto de los ingredientes, no obstante, se reitera, que todavía no se han realizado las suficientes investigaciones sobre el tema. Aunque, en varias investigaciones recientes se evidenció que los efectos positivos relacionados con la cafeína podrían ser mitigados por el componente de la taurina cuando se agrega en una misma bebida [14] 


\subsection{Factores que conllevan al consumo de analépticos y bebidas energizantes}

Se pueden atribuir al consumo de analépticos y bebidas energizantes a factores sociales, a los actos violentos a los que el estudiante se ve expuesto, a los contextos familiares y sociales en los que está inmerso en su diario vivir, lo cual se vincula directamente con el desconocimiento de los posibles factores de riesgo y protección a los que están expuestos al consumir analépticos y bebidas energizantes [15].

Los estudiantes comienzan a consumir analépticos y bebidas energizantes por varias razones que le ayudan a sentirse bien con sentimientos de confianza, poder, relajación y satisfacción. Por lo general los estudiantes consumen estas sustancias cuando sienten estrés y depresión llevándolos a la dependencia de estas.

Por el contrario, hay algunos estudiantes que las ingieren para desempeñarse mejor en diferentes actividades sintiéndose presionados por aumentar o mejorar sus capacidades cognitivas o su rendimiento en alguna disciplina deportiva, siendo esto causante de la experiencia inicial y el abuso por parte de los estudiantes. Otro factor es la curiosidad del estudiante con la justificación: 'porque otros lo hacen' por este motivo los estudiantes son los más vulnerables, debido a la influencia de su ambiente social, el grupo de amigos al cual pertenece [16].

En consecuencia, los estudiantes que no han probado nunca estas sustancias lo hacen por el afán de que sean aceptados en su contexto social, una vez que las prueban es difícil que dejen de consumir. Existen factores sociales y personales que influyen en el consumo.

\subsubsection{Factores sociales}

En estos factores influyen en el estudiante de una manera externa como la publicidad que tienen los medicamentos, sugerencia de amigos o familiares para automedicarse sin contar con la prescripción médica, las influencias en la automedicación como una recomendación o una antigua prescripción, la oferta y disponibilidad con que se encuentran estas sustancias. Otros factores también pueden ser el ciclo de enseñanza que cursa el estudiante, la cuidad de donde provienen y el nivel de ingresos que recibe el estudiante [17].

\subsubsection{Factores personales}

Los factores personales o individuales que influyen en el consumo de analépticos y bebidas energizantes en los estudiantes, se pueden determinar como la baja autoestima, alto nivel de inseguridad, baja tolerancia a la frustración, y rebeldía. El trastorno de 
personalidad es una variable permanente en el comportamiento de cualquier persona factor que puede aumentar el desarrollo de una adicción, por lo cual es muy importante conocer para poder asumir la prevención y tratamiento.

La familia tiene una función indispensable mediante la comunicación con el estudiante como un factor influyente para proporcionarle un contexto familiar seguro, de tal forma que se involucre en las relaciones sociales que mantiene el estudiante, ayudarle a diferenciar las personas que le apoyarán en su desarrollo personal [18].

Los problemas familiares como la falta de relaciones comunicacionales y de afecto pueden aportar a I consumo de estas sustancias, es mejor desarrollar con el estudiante una relación positiva, cálida, protectora, amigable y de confianza para que se compartan dudas y responsabilidades mutuas que coadyuven a y manejar el estrés de manera eficaz que es una de las causas importantes a la hora de consumir las sustancias. Se puede considerar que los núcleos más esenciales que influyen en los estudiantes en este aspecto pueden ser la desintegración familiar, la influencia del medio institucional, la falta de comunicación interpersonal y el desconocimiento de los efectos negativos de estas sustancias.

La Organización Panamericana de la Salud, señala en su informe sobre el desarrollo mundial presentado por el Banco Mundial en 1993, que dentro del 8,1\% de la discapacidad por problemas de salud mental, medida en porcentaje de años de vida ajustados por discapacidad (AVAD) los problemas por dependencia al alcohol y fármacos aportan un

$12,1 \%$ y $4,8 \%$ respectivamente. Acerca del alcohol, explica que se estima un porcentaje del 15 y el $20 \%$ de la población adulta de América Latina como alcohólica o bebedora excesiva. En un estudio realizado en Ecuador, se retoma el concepto de factores de riesgo especialmente en lo que respecta a la 'operatividad para delimitar elementos, circunstancias o hechos que tienen una alta probabilidad en el consumo de droga... como también aquellas circunstancias personales y sociales que relacionadas con las drogas, aumentan la probabilidad de que un sujeto se inicie en el consumo' [19].

\subsubsection{Dependencia al uso de analépticos}

El consumo de sustancias analépticas se considerada perjudicial para la salud debido a los efectos adversos que producen en el organismo. Si bien los individuos que consumen de vez en cuando uno de estos fármacos no son adictos, pero el consumo ocasional suele ser el principio que conduce al individuo a la adicción. Existen 3 estadios para esta secuencia:

Primero, el uso cuando la ingesta de estas sustancias es de manera ocasional, lo cual no genera una dependencia en las personas y sus problemas de salud; el segundo 
consiste en el abuso de estas sustancias, el sujeto se vuelve compulsivo frente a la necesidad de su consumo, su estilo de vida gira en torno a esa necesidad en particular. El último estadio es la dependencia que se puede dar de manera imprevista o progresiva según sea la sustancia que este consumiendo, situación que hace que entre en un círculo vicioso del consumo.

La pretensión de consumir se vuelve para la persona irresistible, provocando que sienta una fuerte ansiedad que solo alivia al consumir de nuevo la sustancia. Hay dos tipos de dependencia: Física y psicológica. La física implica un cambio permanente en el funcionamiento del cuerpo y del cerebro, aquí la droga ya se ha incorporado al metabolismo de la persona, ya se ha generado una tolerancia hacia la sustancia, por lo tanto, el cuerpo cada vez necesita una dosis mayor para sentir los efectos deseados. Al dejar de consumir el cuerpo reacciona con síndromes de abstinencia o retirada. En cambio, la psicológica se da cuando hay una presencia compulsiva para consumir periódicamente la sustancia para experimentar un estado positivo de placer, bienestar y sociabilidad, pero también se consume para redimirse de los estados negativos como el aburrimiento, timidez y estrés [20].

\section{Conclusiones}

Según el análisis de los resultados obtenidos respecto al consumo de los analépticos, el grupo de fármacos que pertenecen a la categoría de efedrinas estimulantes del sistema simpático, son los más consumidas en al menos una vez por semana en un porcentaje mayor por la población femenina que por la masculina de primero a tercer nivel. Es decir que 2 de cada 10 mujeres han consumido este tipo de estimulantes, mientras que en los hombres no hay tanta incidencia.

Los factores personales o sociales de los estudiantes no son causa de influencia en el momento de la elección y decisión, de acuerdo con los resultados obtenidos la gran mayoría de la población estudiada, tanto del sexo femenino como del masculino indicó no tener problemas personales que afecten su rendimiento académico y los lleven a consumir algún estimulante.

Los estudiantes encuestados manifiestan que la causa fundamental para el consumo de analépticos y bebidas energizantes se debe a la falta de organización del tiempo en las actividades que deben cumplir como estudiantes de la carrera de medicina, más que a la influencia social o personal, por ello tanto el sexo masculino como el sexo femenino optan por la alternativa del manejo de 'Medicnopoli', un juego recreacional que brinda información básica sobre psicoanalépticos y energizantes que les permita relajar al menos un periodo de tiempo corto su sistema nervioso central realizando actividades fuera del campo de estudio. 
Del análisis realizado es preocupante observar que muchos estudiantes admiten el ingreso de estas sustancias a su cuerpo sin considerar efectos secundarios que ocasionan y en la mayoría de los casos, no constituyen un impedimento para el consumo, por lo que se puede manifestar que la presión de su carga académica y la preparación de sus exámenes se convierte en el principal motivo para el consumo de los fármacos y bebidas.

Se mencione en la mayoría de casos que las dosis utilizadas suelen ser menos frecuentes, pero se debe tener en cuenta que pueden ser tan adictivas como las drogas y los riesgos que presentan para la salud puede ser altos aún más si son auto medicados y no se cuenta con la recomendación de un profesional.

En la conveniencia de mantener informados a los estudiantes se debe elaborar trípticos sobre los estimulantes mencionados y como evitar su uso al disponer de un organizador de tiempo para cada actividad observando un modelo de horario.

\section{Limitación de Responsabilidad}

Los puntos de vista expresados en el presente trabajo son de entera responsabilidad de las autoras y no de la Escuela Superior Politécnica de Chimborazo, institución en la que prestan los servicios como profesionales de la docencia.

\section{Fuentes de Apoyo}

Para el desarrollo del trabajo investigativo no se contó con fuentes de financiamiento externo, se realizó con el autofinanciamiento de las autoras, si se contó con el apoyo de los estudiantes del tercer nivel de la carrera de medicina, mismos que se encargaron de la recopilación de los datos mediante la aplicación de las encuestas, el procesamiento de la información como aplicación de los conocimientos recibidos en la asignatura de Bioestadística.

\section{Conflicto de Intereses}

Las autoras del trabajo declaran no tener conflicto de interés en la realización, resultados y veracidad de la información.

\section{References}

[1] Tema-11-Christian-2012-13.pdf. 2013. Disponible en: http://www.inspiracle.es/ wp-content/uploads/2013/03/Tema-11-Christian-2012-13.pdf 
[2] Tipos de psicoestimulantes (o psicoanalépticos). Disponible en: https:// psicologiaymente.com/psicofarmacologia/tipos-psicoestimulantes.

[3] Anfetamina - qué es, tipos y cuáles son sus efectos. Recursos de Autoayuda; 2017.

[4] Auge, caída y resurgimiento de las anfetaminas. ABC; 2015. [citado 30 de enero de 2019]. Disponible en: https://www.abc.es/archivo/20150703/ abci-auge-caida-anfetaminas-201506301843.html

[5] Aspectos farmacológicos de las anfetaminas (Pharmacological Aspect of Amphetamines). YouScribe. [Internet]. [Citado 30 de enero de 2019]. Disponible en: https:/www.youscribe.com/catalogue/documents/autres/ aspectos-farmacologicos-de-las-anfetaminas-pharmacological-aspect-2005911

[6] Medicamentos antidepresivos. EcuRed. Disponible en: https://www.ecured.cu/ Medicamentos_antidepresivos

[7] Medicamento - Anticolinérgicos. Para que se toma y efectos secundarios/ Saludemia. Disponible en: https://www.saludemia.com/-/ medicamento-anticolinergicos

[8] Arguedas G, Garnier M, Hong WW, Zaray MC, Rodríguez G. Aspectos médico- legales de los patrones de consumo de bebidas energéticas por parte de los estudiantes de medicina de segundo año de la Universidad de Costa. Med Leg Costa Rica. 2012; 29(1):23-33.

[9] Cote-Menéndez M, Rangel-Garzón CX, Sánchez-Torres MY, Medina-Lemus A. Energy drinks: Rehydrating agents or stimulants? Rev Fac Med. 2011;59(3):255-66.

[10] 232642.pdf [Internet]. [Citado 21 de enero de 2019]. Disponible en: http://riul. unanleon.edu.ni:8080/jspui/bitstream/123456789/5591/1/232642.pdf

[11] Manrique C, Arroyave-Hoyos C, Universidad CES, Galvis-Pareja D. Bebidas cafeínadas energizantes: efectos neurológicos y cardiovasculares. IATREIA. 2018;31(1):6575.

[12] Sánchez JC, Romero CR, Arroyave CD, García AM, Giraldo FD, Sánchez LV. Bebidas energizantes: efectos benéficos y perjudiciales para la salud. Perspect En Nutr Humana. 2015;17(1):79-91.

[13] Guerri M, Psicoactiva P. Las anfetaminas, usos terapéuticos y sus efectos secundarios. Blog de Psicoactiva. 2016 [citado 22 de enero de 2019]. Disponible en: https://www.psicoactiva.com/blog/ las-anfetaminas-usos-terapeuticos-efectos-secundarios/

[14] Ramón-Salvador DM, Cámara-Flores JM, Cabral-León FJ, Juárez-Rojop IE, DíazZagoya JC. Consumo de bebidas energéticas en una población de estudiantes universitarios del estado de Tabasco, México. 2013;19(1):6.

[15] Blanco LE. 2017. Disponible en: https://repositorio.udes.edu.co/bitstream/001/664/ 1/Factorespsicosocialesasociadosalconsumodesustanciaspsicoactivasenestudiant 
esdelaFacultaddeCienciasdelaSaluddelaUniversidadDeSantanderUDES.Bucarama nga.pdf

[16] Nisthal AJG. Consumo de bebidas energéticas en estudiantes internos y médicos residentes. 81.

[17] Behavior of self- medication in students of the Chemistry and Pharmacy career of the Universidad Católica del Norte. ResearchGate. [citado 23 de enero de 2019]. Disponible en: https://www.researchgate.net/publication/326635279_ Comportamiento_de_la_automedicacion_en_estudiantes_de_la_carrera_de_ Quimica_y_Farmacia_de_la_Universidad_Catolica_del_Norte_Behavior_of_ self-medication_in_students_of_the_Chemistry_and_Pharmacy_career

[18] Hermida JRF, Rodríguez OG, Fernández GG, Artamendi SF. Normas de publicación. :56.

[19] Rojas CEC, Mora LPL. Una estrategia educativa para disminuir riesgo de consumo de sustancias psicoactivas en estudiantes de séptimo grado.:236.

[20] Trabajo de Titulación [Internet]. [citado 23 de enero de 2019]. Disponible en: $\quad$ http://dspace.ucuenca.edu.ec/jspui/bitstream/123456789/28413/1/ Trabajo\{\%\}20de\{\%\}20Titulacion.pdf

[21] Global Food Safety. ¿Qué son las bebidas energizantes? Disponible en: http://www. gfs.com.pe/noticias/que-son-bebidas-energizantes.html

[22] Sánchez JC, Romero CR, Arroyave CD et al. Bebidas energizantes: efectos benéficos y perjudiciales para la salud. Perspect En Nutr Humana. 2015;17(1).

[23] Uso indebido de drogas en estudiantes de 6to. Año de medicina [Internet]. [citado 24 de enero de 2019]. Disponible en: http://bvs.sld.cu/revistas/rhab/vol4_num5/uso_ indebido_de_drogas.htm

[24] Carrión DG, Illingworth GV, Cevallos JP et al. Plan nacional de prevención integral de drogas 2012 - 2013.:93.

[25] RIA. Consumo de bebidas energéticas en estudiantes universitarios. RIA. Disponible en: https://ria.asturias.es/RIA/handle/123456789/7025

[26] Sanchis-Gomar F, Pareja-Galeano H, Cervellin G, Lippi G, Earnest CP. Energy drink overconsumption in adolescents: Implications for arrhythmias and other cardiovascular events. The Canadian Journal of Cardiology. 2015;31(5):572-575.

[27] Reissig CJ, Strain EC, Griffiths RR. Caffeinated Energy Drinks - A Growing Problem. Drug and alcohol dependence, 2009;99(1-3):1- 10.

[28] CARIM-Roemmers. PR Vademecum Uruguay. 2015. Available from: http://uy. prvademecum.com/producto.php?producto $=7237$

[29] El nuevo abuso: pastillas para mantenerse despierto. La nación. 2010. [cited 2019 Jan 26]. Available from: https://www.lanacion.com.ar/ 1279060-el-nuevo-abuso-pastillas-para-mantenerse-despierto 
[30] Ritalin, la pastilla de la \&quot;concentración\&quot. BBC MUNDO. 2016. Available from: https://www.bbc.com/mundo/noticias-36482882 